Publ. RIMS, Kyoto Univ.

22 (1986), 31-42

\title{
Stationary Fourier Hyperprocesses
}

By

\author{
Yoshifumi ITO*
}

\section{Introduction}

In this paper, we will define stationary Fourier hyperprocesses as an extension of stationary random functions and stationary random distributions in a similar way to Itô [6] and study their properties.

In $\S 1$, we will first introduce some fundamental notions and prepare some notations.

In $\S 2$, we will define the covariance Fourier hyperfunctions of stationary Fourier hyperprocesses, which correspond to Khintchine's covariance functions and Itô's covariance distributions [15], [6].

In $\S 3$, we will prove the spectral decomposition theorem of covariance Fourier hyperfunctions.

In $\S 4$, we will prove the spectral decomposition theorem of stationary Fourier hyperprocesses.

In $\S 5$, we will mention the derivatives of stationary Fourier hyperprocesses.

\section{§. Fundamental Notions and Notations}

In this paper we will restrict ourselves to complex valued random variables with mean 0 and finite variance. Let $H$ be the Hilbert space constituted by all such variables. In $H$, we define the inner product by the following relation:

$$
(X, Y)=E(X \cdot \bar{Y}), \quad \text { for } \quad X, Y \in H,
$$

where $E$ denotes the expectation. We will here consider only the strong topology on $H$. A continuous random process $X(t),-\infty<t<\infty$, is an $H$-valued continuous function on $\boldsymbol{R}=(-\infty, \infty)$. The set of all continuous processes will be denoted by $C(H)$.

Now we will remember the notions of Fourier hyperfunctions and vector valued Fourier hyperfunctions following Sato [17], Kawai [13], [14], Ito and

Communicated by M. Kashiwara, June 6, 1985.

* Department of Mathematics, Tokushima University, Tokushima, Japan.

** This work was partially supported by the Grant in Aid for Scientific Research (No. 60540113). 
Nagamachi [9], [10], Junker [11], [12], Ito [7], [8]. Let $\boldsymbol{D}=[-\infty, \infty]$ be a directional compactification of $\boldsymbol{R}=(-\infty, \infty)$. Let $\mathcal{A}$ be the sheaf of germs of rapidly decreasing real analytic functions over $\boldsymbol{D}$. Let $\underset{\sim}{A}=\mathcal{A}(\boldsymbol{D})$ be the space of all sections of $\mathcal{A}$ on $\boldsymbol{D}$. $\underset{\sim}{A}$ is endowed with the usual DFS topology. A Fourier analytic functional defined on $\underset{\sim}{A}$ is called a Fourier hyperfunction on $\boldsymbol{D}$ and a Fourier analytic linear mapping from $\underset{\sim}{A}$ to $H$ is called an $H$-valued Fourier hyperfunction on $\boldsymbol{D}$. We will denote by $A^{\prime}$ the space of all Fourier hyperfunctions on $\boldsymbol{D}$ and by $A^{\prime}(H)$ the space of all $H$-valued Fourier hyperfunctions on $\boldsymbol{D}$.

Definition 1.1. A Fourier hyperprocess is defined to be an $H$-valued Fourier hyperfunction.

Remark. Our concept of Fourier hyperprocesses is a generalization of Okabe's concept of hyperprocesses in [18], Definition 6.1.

Let $\tilde{\boldsymbol{C}}(H)$ be the set of all $H$-valued continuous functions on $\boldsymbol{R}$ which satisfy the following estimate:

$$
\text { for any } \varepsilon>0, \sup \left\{\|X(t)\| e^{-\varepsilon|t|} ; t \in \boldsymbol{R}\right\}<\infty \text {, }
$$

where \|\| denotes the norm on $H$. An element of $\tilde{\mathcal{C}}(H)$ is called a slowly increasing continuous process. Then $\tilde{\boldsymbol{C}}(H)$ may be considered as a subsystem of $A^{\prime}(H)$, since we can identify a slowly increasing continuous random process $X(t)$ with the following Fourier hyperprocess $X(\phi)$ determined by it:

$$
X(\phi)=\int X(t) \phi(t) d t \equiv \int_{-\infty}^{\infty} X(t) \phi(t) d t, \quad \text { for } \quad \phi \in A
$$

The following notations will be often used in the theory of Fourier hyperfunctions. Let $F \in A^{\prime}$ or $A_{\sim}^{\prime}(H)$ and $\phi \in A$.

$\tau_{h}$ (shift transformation): $\tau_{h} \phi(t)=\phi(t+h), \tau_{h} F(\phi)=F\left(\tau_{-h} \phi\right)$.

$D$ (derivative) $: D \phi(t)=\phi^{\prime}(t), D F(\phi)=-F(D \phi)$.

$\checkmark$ (inversion) : $\check{\phi}(t)=\phi(-t), \breve{F}(\phi)=F(\check{\phi})$.

- (conjugate): $\bar{\phi}(t)=\overline{\phi(t)}, \bar{F}(\phi)=\overline{F(\bar{\phi})}$.

$\sim(=\bar{v}=\underline{ }): \tilde{\phi}(t)=\overline{\phi(-t)}, \tilde{F}(\phi)=\overline{F(\tilde{\phi})}$.

^ (Fourier transformation): $\hat{\phi}(\lambda)=\int e^{-i 2 \pi \lambda t} \phi(t) d t, \hat{F}(\phi)=F(\hat{\phi})$.

The following relation should be noted.

$$
(F * \phi)(0)=F(\check{\phi})=\breve{F}(\phi), \quad(\phi * \psi)^{\wedge}=\hat{\phi} \cdot \hat{\psi}, \hat{\tilde{\phi}}=\overline{\hat{\phi}} .
$$

Generalizing Khintchine-Itô's notions of (weakly) stationary processes, we have the following 
Definition 1.2. We will call $X \in A_{\sim}^{\prime}(H)$ weakly stationary or merely stationary for short if we have, for any $\phi, \phi \in \underset{\sim}{A}$,

$$
\left(\tau_{h} X(\phi), \tau_{h} X(\psi)\right)=(X(\phi), X(\psi))
$$

and strictly stationary if the joint probability law of

$$
\left(\tau_{h} X\left(\phi_{1}\right), \cdots, \tau_{h} X\left(\phi_{n}\right)\right)
$$

is independent of $h$ for any $n$ and $\phi_{1}, \cdots, \phi_{n} \in A$.

We shall adopt here the following notations:

$S$ : the totality of stationary Fourier hyperprocesses,

$S^{0}$ : the totality of slowly increasing stationary processes,

$\bar{S}$ : the totality of strictly stationary Fourier hyperprocesses,

$\bar{S}^{0}$ : the totality of slowly increasing strictly stationary processes.

Clearly we have

$$
S \supset \bar{S} \cup S^{0}, \quad S^{0} \supset \bar{S}^{0} .
$$

Definition 1.3. A Fourier hyperprocess $X$ is called a complex normal Fourier hyperprocess if $X(\phi), \phi \in A$, constitute a complex normal system and a real normal Fourier hyperprocess if $X$ is real viz. $X=\bar{X}$ and $X(\phi), \phi$ running over real functions in $\underset{\sim}{A}$, constitute a (real) normal system (see Itô [4], [5] and Hida [3]).

This is an extension of normal processes or Gaussian processes (Doob [1], II, §3) and complex (or real) normal random distributions (Itô [6]). A (complex as well as real) normal Fourier hyperprocess will be strictly stationary, if it is weakly stationary. The corresponding fact is well-known regarding stationary processes.

We shall here mention a typical example of real stationary Fourier hyperprocesses which are not stationary processes. Let $B(t)$ be a (real) Brownian motion process (Doob [1], p. 97). The derivative (in the sense of Fourier hyperfunctions) of this process $B^{\prime} \equiv D B$ is a Fourier hyperprocess defined by

$$
B^{\prime}(\phi)=-B\left(\phi^{\prime}\right)=\int \phi(t) d B(t)
$$

(Wiener integral, see Itô [4]). This is evidently real normal and stationary, since

$$
\begin{aligned}
& \left(\tau_{h} B^{\prime}(\phi), \tau_{h} B^{\prime}(\psi)\right)=\left(B^{\prime}\left(\tau_{-h} \phi\right), B^{\prime}\left(\tau_{-h} \psi\right)\right) \\
& =\left(\int \phi(t-h) d B(t), \int \phi(t-h) d B(t)\right) \\
& =\int \phi(t-h) \overline{\psi(t-h)} d t=\int \phi(t) \overline{\psi(t)} d t
\end{aligned}
$$


which shows that $B^{\prime} \in S$. The fact that $B^{\prime} \notin S^{0}$ will be proved in $\S 2$.

\section{§ 2. Covariance Fourier Hyperfunctions}

Similarly to Khintchine-Itô's notion of covariance distributions, we will here define the notion of the covariance Fourier hyperfunctions.

Theorem 2.1. Let $X(\phi)$ be any stationary Fourier hyperprocess. Then there exists one and only one Fourier hyperfunction $\rho \in A^{\prime}$ satisfying the relation

$$
(X(\phi), X(\phi))=\rho(\phi * \tilde{\psi}), \quad \phi, \phi \in A .
$$

Definition 2.2. The Fourier hyperfunction $\rho$ in Theorem 2.1 is called the covariance Fourier hyperfunction of $X$.

Proof of Theorem 2.1. If we put

$$
T_{\phi}(\phi)=(X(\phi), X(\bar{\psi})), \quad \phi, \phi \in \underset{\sim}{A},
$$

then we get a Fourier hyperfunction $T_{\phi} \in A^{\prime}$ for each $\phi \in A$. Taking into account the fact that $T_{\phi}(\psi)$ is continuous in $(\phi, \phi) \in A \times \underset{\sim}{A}$ and by virtue of Kernel Theorem, we will easily see that $\phi \rightarrow T_{\phi}$ is a continuous linear mapping from $\underset{\sim}{A}$ into $A_{\sim}^{\prime}$ (see Grothendieck [2], Chap. II, Théorèm 12 and Ito [7]). Furthermore this transformation commutes with the shift transformation:

$$
\begin{aligned}
\left(\tau_{h} T_{\dot{\phi}}\right)(\phi) & =T_{\phi}\left(\tau_{-h} \psi\right)=\left(X(\phi), X\left(\overline{\tau_{-h} \phi}\right)\right) \\
& =\left(X(\phi), X\left(\tau_{-h} \bar{\psi}\right)\right)=\left(X\left(\tau_{h} \phi\right), X(\bar{\psi})\right) \\
& =T_{\tau_{h} \phi}(\phi) .
\end{aligned}
$$

Here we will use the following

Lemma. A continuous linear mapping $\phi \rightarrow T_{\phi}$ from $\underset{\sim}{A}$ to $A_{\sim}^{\prime}$ commutes with the shift transformation if and only if there exists a Fourier hyperfunction $T$ such that $T_{\phi}=T * \phi$ holds.

Postponing the proof of this Lemma until the end of the proof of this Theorem, we will continue the proof of the Theorem. Thus by the above Lemma $T_{\phi}$ is expressible as a convolution of a Fourier hyperfunction $T$ and $\phi$ :

Hence it follows that

$$
T_{\phi}=T * \phi
$$

$$
\begin{aligned}
(X(\phi), X(\phi)) & =T_{\phi}(\bar{\psi})=(T * \phi)(\bar{\psi}) \\
& =(T * \phi * \tilde{\psi})(0)=\rho(\phi * \tilde{\psi}),
\end{aligned}
$$

where we put $\rho=\check{T}$.

The uniqueness of $\rho$ follows at once from the fact that the set of all 
elements of the form $\phi * \phi, \phi, \phi \in \underset{\sim}{A}$, is dense in $\underset{\sim}{A}$.

Q.E.D.

Proof of Lemma. It is evident that a continuous linear mapping $\phi \rightarrow T_{\phi}=$ $T * \phi$ commutes with the shift transformation. Thus we have only to prove that $T_{\phi}$ is expressible as a convolution of a Fourier hyperfunction $T$ and $\phi: T_{\phi}=T * \phi$ if it commutes with the shift transformation.

We will first show that, if $\alpha \in \underset{\sim}{A}$, we have

$$
T_{a * \phi}=T_{\alpha} * \phi \text {. }
$$

In fact, we have, by the definition of integration,

$$
\begin{aligned}
\langle\phi, \phi\rangle & =\int \phi(t) \phi(t) d t \\
& =\lim _{\jmath} \sum_{n} a_{n \jmath} \psi\left(h_{n \jmath}\right) \\
& =\left\langle\lim _{\jmath} \sum_{n} a_{n \jmath} \tau_{h_{n \jmath}} \delta, \phi\right\rangle
\end{aligned}
$$

for any $\phi \in \underset{\sim}{A}$ considering $\phi$ as a Fourier hyperfunction, where $\sum_{n}$ 's are finite sums. Thus we have the relation

$$
\phi=\lim _{j} \sum_{n} a_{n j} \tau_{h_{n j}} \delta
$$

as a Fourier hyperfunction. Therefore we have

$$
\begin{aligned}
\alpha * \phi(t) & =\left(\lim _{\jmath} \sum_{n} a_{n,} \tau_{h_{n j}} \delta\right) * \alpha(t) \\
& =\lim _{\jmath} \sum_{n} a_{n \jmath} \tau_{h_{n \jmath}} \alpha(t) .
\end{aligned}
$$

Hence we have, by the assumption,

$$
\begin{aligned}
T_{\alpha k \phi} & =\lim _{\jmath} \sum_{n} a_{n \jmath} \tau_{h_{n \jmath}} T_{\alpha} \\
& =\left(\lim _{\jmath} \sum_{n} a_{n \jmath} \tau_{h_{n \jmath}} \delta\right) * T_{\alpha} \\
& =\phi * T_{\alpha}=T_{a} * \phi .
\end{aligned}
$$

Then if we choose a sequence $\alpha_{\nu} \in \underset{\sim}{A}$ which converges to $\delta$ in $A_{\sim}^{\prime}$, the sequence $\alpha_{\nu} * \phi$ converges to $\phi$ in $\underset{\sim}{A}$. Hence $T_{\alpha_{\nu^{*} \phi}}$ converges to $T_{\phi}$ in $A^{\prime}$. Hence, for the Fourier hyperfunctions $T_{\alpha_{\nu}}$, their regularizations $T_{\alpha_{\nu}} * \phi$ converges in $\underset{\sim}{A^{\prime}}$ for every $\phi \in \underset{\sim}{A}$. Thus $T_{\alpha_{\nu}}$ itself converges in $\underset{\sim}{A}$. If $T$ is its limit, we have

$$
\begin{aligned}
& T_{\phi}=T * \phi, \quad T \in A^{\prime} . \\
& \text { Q.E.D. }
\end{aligned}
$$

Theorem 2.3. If $X(\phi)$ is a real stationary Fourier hyperprocess, then its covariance Fourier hyperfunction is real, i.e. $\rho=\bar{\rho}$.

Proof. Let $\rho$ be the covariance Fourier hyperfunction. Then that of $\bar{X}$ 
will become $\bar{\rho}$, since we have

$$
\begin{aligned}
& (\bar{X}(\phi), \bar{X}(\phi))=(\overline{X(\bar{\phi})}, \overline{X(\bar{\psi})})=(\overline{X(\bar{\phi}), X(\bar{\phi})}) \\
& =\overline{\rho(\bar{\phi} * \tilde{\bar{\psi}})}=\overline{\rho(\overline{\phi * \tilde{\phi}})} \\
& =\bar{\rho}(\phi * \tilde{\psi}) \text {. }
\end{aligned}
$$

Thus $X=\bar{X}$ implies $\rho=\bar{\rho}$.

Q.E.D.

Example. The covariance Fourier hyperfunction of $B^{\prime}$ is Dirac's $\delta$-function, since

$$
\begin{aligned}
\left(B^{\prime}(\phi), B^{\prime}(\psi)\right) & =\int \phi(t) \overline{\psi(t)} d t=\int \phi(t) \tilde{\psi}(-t) d t \\
& =(\phi * \tilde{\psi})(0)=\delta(\phi * \tilde{\psi})
\end{aligned}
$$

Thus we see that $B^{\prime} \oplus S^{0}$, because, if $B^{\prime} \in S^{0}$, then the covariance Fourier hyperfunction would be induced by a slowly increasing continuous function as shown similarly to Itô [6], §2.

\section{$\S 3$. Spectral Decomposition of Covariance Fourier Hyperfunctions}

Let $X(\phi)$ be any stationary Fourier hyperprocess with the covariance Fourier hyperfunction $\rho$. Then we have

$$
\rho(\phi * \tilde{\phi})=(X(\phi), X(\phi)) \geqq 0,
$$

which implies that $\rho$ is a positive semidefinite Fourier hyperfunction. Thus, by virtue of Bochner-Nagamachi-Mugibayashi-Junker's Theorem (see NagamachiMugibayashi [16], Theorem 4.1 and Junker [12], Theorem 5.8), we have the following

Theorem 3.1. $\rho$ is expressible in the form

$$
\rho(\phi)=\int \hat{\phi}(\lambda) d \mu(\lambda), \quad \phi \in \underset{\sim}{A}
$$

in one and only one way, where $\mu$ is a nonnegative measure satisfying

$$
\int e^{-\varepsilon|\lambda|} d \mu(\lambda)<\infty
$$

for every $\varepsilon>0$.

Definition 3.2. We will call the expression $(*)$ the spectral decomposition of $\rho$ and $\mu$ the spectral measure of $\rho$.

Conversely we have

Theorem 3.3. Any Fourier hyperfunction of the above form (*) is the 
covariance Fourier hyperfunction of a stationary Fourier hyperprocess which is complex normal.

Proof. Let $\rho$ be a Fourier hyperfunction of the above form. Put

$$
\Gamma(\phi, \phi)=\rho(\phi * \tilde{\psi}), \quad \phi, \phi \in \underset{\sim}{A} .
$$

Then $\Gamma(\phi, \phi)$ is positive semidefinite in $(\phi, \phi)$, as we have

$$
\sum_{i, j=1}^{n} \Gamma\left(\phi_{i}, \phi_{j}\right) \xi_{i} \bar{\xi}_{\jmath}=\rho(\theta * \tilde{\theta}) \geqq 0, \quad \theta=\sum_{i} \xi_{2} \phi_{i}
$$

Therefore we can define a complex normal system $X(\phi), \phi \in \underset{\sim}{A}$, such that $E X(\phi)=0$ and $E(X(\phi) \cdot \overline{X(\phi)})=\Gamma(\phi, \phi)=\rho(\phi * \tilde{\psi})$ as in Itô [5] and Hida [3]. It remains only to show that $X(\phi)$ is a Fourier hyperprocess. From the identity:

$$
\begin{aligned}
\|X(c \phi)-c X(\phi)\|^{2}= & (X(c \phi), X(c \phi))-c(X(\phi), X(c \phi)) \\
& -\bar{c}(X(c \phi), X(\phi))+c \bar{c}(X(\phi), X(\phi)) \\
= & \rho(c \phi * \widetilde{c \phi})-c \rho(\phi * \widetilde{\phi \phi})-\bar{c} \rho(c \phi * \tilde{\phi})+c \bar{c} \rho(\phi * \tilde{\phi}) \\
= & c \bar{c} \rho(\phi * \tilde{\phi})-c \bar{c} \rho(\phi * \tilde{\phi})-c \bar{c} \rho(\phi * \tilde{\phi})+c \bar{c} \rho(\phi * \tilde{\phi}) \\
= & 0,
\end{aligned}
$$

it follows that $X(c \phi)=c X(\phi)$. By a similar way we can see that $X(\phi+\phi)=$ $X(\phi)+X(\psi)$. Therefore $X(\phi)$ is linear in $\phi$. By the identity $\|X(\phi)\|^{2}=\rho(\phi * \tilde{\phi})$ we obtain the continuity of $X$. Thus our theorem is completely proved.

Q.E.D.

Next we shall discuss the case of real stationary Fourier hyperprocesses. By Theorem 2.3 we see that $\rho=\bar{\rho}$ in this case. But we have

$$
\begin{aligned}
& \bar{\rho}(\phi)=\overline{\rho(\bar{\phi})}=\int \overline{\hat{\phi}}(\lambda) d \mu(\lambda)=\int \hat{\phi}(-\lambda) d \mu(\lambda)=\int \hat{\phi}(\lambda) d \check{\mu}(\lambda) \\
& (\check{\mu}(E)=\mu(-E),-E=\{t ;-t \in E\}) .
\end{aligned}
$$

By the uniqueness of the spectral measure we will obtain the following

Theorem 3.4. In the case of a real stationary Fourier hyperprocess, the spectral measure $\mu$ is symmetric with respect to 0 , viz. $\mu(E)=\mu(-E)$.

Conversely we have

Theorem 3.5. Any Fourier hyperfunction of the form (*) with a symmetric measure $\mu$ is the covariance Fourier hyperfunction of a certain stationary Fourier hyperprocess which is real normal.

The proof is similar to that of Theorem 3.3; we use the existence theorem of real normal systems instead of complex normal ones. 
Example. $B^{\prime}$ is a real stationary Fourier hyperprocess whose spectral measure is the ordinary Lebesgue measure, because we have

$$
\delta(\phi)=\phi(0)=\int \hat{\phi}(\lambda) d \lambda .
$$

\section{$\S 4$. Spectral Decomposition of Stationary Fourier Hyperprocesses}

We will first introduce a random hypomeasure. Let $\mu$ be a nonnegative measure defined for all Borel sets in $\boldsymbol{R}$ and $\boldsymbol{B}^{*}$ denote the system of all Borel sets with finite $\mu$-measure.

Definition 4.1. An $H$-valued function $M(E)$ defined for $E \in \boldsymbol{B}^{*}$ is called a random hypomeasure with respect to $\mu$ if

holds.

$$
\left(M\left(E_{1}\right), M\left(E_{2}\right)\right)=\mu\left(E_{1} \cap E_{2}\right), \quad E_{1}, E_{2} \in B^{*}
$$

We get, by the definition,

Theorem 4.2. Let $M(E)$ be a random hypomeasure with respect to $\mu$. Then we have

(1) $\|M(E)\|^{2}=\mu(E)$,

(2) $M\left(E_{1}\right) \perp M\left(E_{2}\right)$ if $E_{1} \cap E_{2}=\varnothing$,

(3) If $E_{1}, E_{2}, \cdots$ are disjoint to each other and belong to $\boldsymbol{B}^{*}$ with their sum $E=\sum_{n=1}^{\infty} E_{n}, M(E)=\sum_{n=1}^{\infty} M\left(E_{n}\right)$, (in $\left.H\right)$.

We can easily define the integral with respect to the random hypomeasure (Doob [1], IX, §2):

$$
M(f)=\int f(\lambda) d M(\lambda)
$$

for $f \in L^{2}(\boldsymbol{R}, \mu)$.

Then we have the following

Theorem 4.3. Let $M(f)$ be as above. Then we have, for $f_{1}, f_{2} \in L^{2}(\boldsymbol{R}, \mu)$ and $c_{1}, c_{2} \in C$,

(1) $\left(M\left(f_{1}\right), M\left(f_{2}\right)\right)=\left(f_{1}, f_{2}\right)\left(\equiv \int f_{1}(\lambda) \overline{f_{2}(\lambda)} d \mu(\lambda)\right)$,

(2) $M\left(c_{1} f_{1}+c_{2} f_{2}\right)=c_{1} M\left(f_{1}\right)+c_{2} M\left(f_{2}\right)$.

Theorem 4.4. Let $X$ be any stationary Fourier hyperprocess with the spectral measure $\mu$. Then $X(\phi)$ will be expressible in the form

$$
X(\phi)=\int \hat{\phi}(\lambda) d M(\lambda)=M(\hat{\phi})
$$


in one and only one way, $M$ being a random hypomeasure with respect to $\mu$. Conversely, any Fourier hyperprocess of such form is a stationary Fourier hyperprocess.

Definition 4.5. We will call the expression (**) the spectral decomposition of $X$ and $M$ the spectral hypomeasure of $X$.

Proof of Theorem 4.4. We will first remark that $\underset{\sim}{A}$ is dense in $L^{2} \equiv L^{2}(\mathbb{R}, \mu)$. Then the Fourier transformation is a topological isomorphism from $\underset{\sim}{A}$ onto itself. Thus the uniqueness of the expression is clear.

In order to prove the possibility of the expression, we will put

$$
T(\phi)=X(\phi) \quad \text { for } \quad \phi=\hat{\phi} .
$$

Then $T$ will be a mapping from $\underset{\sim}{A}\left(\subset L^{2}\right)$ into $H$, which is clearly linear and isometric on account of the identity:

$$
\begin{aligned}
\|T(\phi)\|^{2} & =(X(\phi), X(\phi))=\rho(\phi * \tilde{\phi}) \\
& =\int|\psi(\lambda)|^{2} d \mu(\lambda)=\|\psi\|^{2},
\end{aligned}
$$

since $(\phi * \tilde{\phi})^{\wedge}=|\hat{\phi}|^{2}$. $\underset{\sim}{A}$ being dense in $L^{2}$, we can extend $T(\psi)$ to a linear isometric mapping from $L^{2}$ into $H$. As the characteristic function $\chi_{E}(\lambda)$ of a set $E \in B^{*}$ belongs to $L^{2}$, we may define $M(E)$ as follows:

Then we have

$$
M(E)=T\left(\chi_{E}\right) .
$$

$$
\left(M\left(E_{1}\right), M\left(E_{2}\right)\right)=\int \chi_{E_{1}}(\lambda) \overline{\chi_{E_{2}}(\lambda)} d \mu(\lambda)=\mu\left(E_{1} \cap E_{2}\right),
$$

since $T$ is isometric. In addition to this, we will have

$$
M(f)=T(f) \quad \text { for } \quad f \in L^{2},
$$

for this is evidently true for any simple function $f$ in $L^{2}$ by the definition and we will easily see that it is also true for any $f \in L^{2}$, by taking into account the fact that both sides of (***) are isometric in $f$ and any $f \in L^{2}$ is expressed as the $L^{2}$-limit of a sequence of simple functions. If we put $f=\hat{\phi}$ in (***), we obtain $(* *)$ at once. The last part of the theorem is clear by the definitions.

Q.E.D.

Making use of this theorem we can characterize the class of slowly increasing stationary processes.

Theorem 4.6. A slowly increasing stationary process $X$ is a stationary Fourier hyperprocess with the spectral measure such that 


$$
\int d \mu(\lambda)<\infty
$$

Proof. A slowly increasing stationary process $X$ is a stationary continuous random process. By Khinchine's Theorem (see Khinchine [15]), it spectral measure $\mu$ satisfies the assumption of the theorem and, by virtue of Doob [1], $\mathrm{XI}, \S 4$, we have the expression

$$
X(\phi)=\int \hat{\phi}(\lambda) d M(\lambda)=M(\hat{\phi}) .
$$

Thus, by Theorem 4.4, $X$ induces a stationary Fourier hyperprocess with the spectral measure $\mu$.

Conversely, let $X$ be a stationary Fourier hyperprocess with the spectral measure $\mu$ which satisfies the assumption of the theorem. Then we have

$$
X(\phi)=\int \hat{\phi}(\lambda) d M(\lambda), \quad\left(M\left(E_{1}\right), M\left(E_{2}\right)\right)=\mu\left(E_{1} \cap E_{2}\right),
$$

where

$$
\int d \mu(\lambda)<\infty
$$

Put

$$
Y(t)=\int e^{-i 2 \pi \lambda t} d M(\lambda),
$$

which may be defined, since the $\lambda$-function $e^{-i 2 \pi \lambda t}$ belongs to $L^{2}$ by virtue of the assumption on $\mu, Y(t)$ proves to be a stationary continuous random process and, what is more, it becomes a slowly increasing stationary continuous random process. Therefore, we have, for $\phi \in \underset{\sim}{A}$,

$$
\begin{aligned}
\int Y(t) \phi(t) d t & =\int \phi(t) \int e^{-i 2 \pi \lambda t} d M(\lambda) d t \\
& =\int \hat{\phi}(\lambda) d M(\lambda)=X(\phi),
\end{aligned}
$$

which implies that $X(\phi)$ is induced by a slowly increasing stationary process $Y$.

Q.E.D.

In the proof of the above theorem, we have the following

Corollary. A slowly increasing stationary continuous random process and a stationary continuous random process are identical.

By the same way as in Theorem 3.4, we will obtain

Theorem 4.7. In the case of a real stationary Fourier hyperprocess the spectral random hypomeasure $M$ is hermitian symmetric, i.e. $M(E)=\overline{M(-E)}$. 


\section{$\S 5$. Derivatives of Stationary Fourier Hyperprocesses}

Any Fourier hyperprocess has derivatives of any order, which are also Fourier hyperprocesses.

Theorem 5.1. Let $X$ be a stationary Fourier hyperprocess with the spectral measure $\mu$ and the spectral random hypomeasure $M$. Then $X^{(k)}\left(=D^{k} X\right)$ is also a stationary Fourier hyperprocess whose spectral measure $\mu_{k}$ and spectral random hypomeasure $M_{k}$ are given by

$$
d \mu_{k}(\lambda)=(2 \pi \lambda)^{2 k} d \mu(\lambda), \quad d M_{k}(\lambda)=(i 2 \pi \lambda)^{k} d M(\lambda) .
$$

Proof. We have, by definition,

$$
\begin{aligned}
X^{(k)}(\phi) & =(-1)^{k} X\left(\phi^{(k)}\right)=(-1)^{k} \int \hat{\phi}^{(k)}(\lambda) d M(\lambda) \\
& =\int(i 2 \pi \lambda)^{k} \phi(\lambda) d M(\lambda)
\end{aligned}
$$

since we have, for $\phi \in \underset{\sim}{A}$,

$$
\hat{\phi}^{(k)}(\lambda)=(-1)^{k}(i 2 \pi \lambda)^{k} \phi(\lambda) .
$$

Thus $X^{(k)}$ proves to be a stationary Fourier hyperprocess satisfying the above conditions.

Q.E.D.

By Theorem 4.6 we have the following

Theorem 5.2. In order that $X^{(k)}$ is a stationary continuous process, it is necessary and sufficient that the spectral measure $\mu$ of $X$ satisfies

$$
\int \lambda^{2 k} d \mu(\lambda)<\infty
$$

\section{References}

[1] Doob, J.L., Stochastic processes, John Wiley \& Sons, New York-London-Sydney, 1953.

[2] Grothendieck, A., Produit tensoriels topologiques et espaces nucléaires, Memoirs of the Amer. Math. Soc., No. 16, American Mathematical Society, 1966.

[3] Hida, T., Brownian motion, Iwanami, Tokyo, 1975 (in Japanese).

[4] Itô, K., Multiple Wiener integral, J. Math. Soc. Japan, 3 (1951), 156-169.

[5] - Complex multiple Wiener integral, Japan. J. Math., 22 (1952), 63-86.

[6] - Stationary random distributions, Memoirs of the College of Science, Univ. of Kyoto, Ser. A, 28 (1953), 209-223.

[7] Ito, Y., Fourier hyperfunctions of general type, 1983, Preprint.

[8] - Theory of (Vector Valued) Fourier Hyperfunctions. Their Realization as Boundary Values of (Vector Valued) Slowly Increasing Holomorphic Functions, (I), J. Math. Tokushima Univ., 18 (1984), 57-101. 
[9] Ito, Y. and Nagamachi, S., Theory of $H$-valued Fourier Hyperfunctions, Proc. Japan Acad., 51 (1975), 558-561.

[10] - On the Theory of Vector Valued Fourier Hyperfunctions, J. Math. Tokushima Univ., 9 (1975), 1-33.

[11] Junker, K., Vektorwertige Fourierhyperfunktionen, Diplomarbeit, Düsseldorf. 1978.

[12] - Vektorwertige Fourierhyperfunktionen und ein Satz von Bochner-ScwartzTyp, Inaugural-Dissertation, Düsseldorf, 1979.

[13] Kawai, T., The theory of Fourier transformations in the theory of hyperfunctions and its applications, Surikaiseki Kenkyusho Kokyuroku, R.I.M.S., Kyoto Univ., 108 (1969), 84-288 (in Japanese).

[14] - On the theory of Fourier hyperfunctions and its applications to partial differential equations with constant coefficients, J. Fac. Sci., Univ. Tokyo, Sec. IA, 17 (1970), 465-517.

[15] Khintchine, A., Korrelationstheorie des stationäre stochastischen Prozesse, Math. Ann., 109 (1934), 604-615.

[16] Nagamachi, S., and Mugibayashi, N., The Haag-Ruelle Formulation of Scattering in Hyperfunction Quantum Field Theory, Reports on Mathematical Physics, 16 (1979), 181-201.

[17] Sato, M., Theory of hyperfunctions, I, J. Fac. Sci., Univ. Tokyo, Sect. I, 8 (1959), 139-193.

[18] Okabe, Y., Stationary Gaussian processes with Markovian property and M. Sato's hyperfunctions, Japanese J. of Math., 41 (1973), 69-122. 